\title{
Médiévales
}

Langues, Textes, Histoire

72 | printemps 2017

Roman du Genji et société aristocratique au Japon

Jean-Louis RocH (dir.), Tabellionages au Moyen Âge en Normandie, un notariat à découvrir

Rouen, Presses universitaires de Rouen et du Havre, 2014 ( Changer d'époque $", n^{\circ} 28$ )

\section{Anne Kucab}

\section{(2) OpenEdition}

1 Journals

\section{Édition électronique}

URL : https://journals.openedition.org/medievales/8135

DOI : 10.4000/medievales.8135

ISSN : $1777-5892$

Éditeur

Presses universitaires de Vincennes

\section{Édition imprimée}

Date de publication : 22 juin 2017

Pagination : 197-199

ISBN : 978-2-84292-612-0

ISSN : 0751-2708

\section{Référence électronique}

Anne Kucab, "Jean-Louis восн (dir.), Tabellionages au Moyen Âge en Normandie, un notariat à découvrir », Médiévales [En ligne], 72 I printemps 2017, mis en ligne le 08 juillet 2017, consulté le 23 avril 2022.

URL : http://journals.openedition.org/medievales/8135; DOI : https://doi.org/10.4000/medievales. 8135

Ce document a été généré automatiquement le 23 avril 2022.

Tous droits réservés 


\section{Jean-Louis ROCH (dir.), Tabellionages au Moyen Âge en Normandie, un notariat à découvrir}

Rouen, Presses universitaires de Rouen et du Havre, 2014 ( Changer d'époque ", $\mathrm{n}^{\circ} 28$ )

\section{Anne Kucab}

\section{RÉFÉRENCE}

Jean-Louis RocH (dir.), Tabellionages au Moyen Âge en Normandie, un notariat à découvrir, Rouen, Presses universitaires de Rouen et du Havre, 2014, 160 p. ("Changer d'époque », $\left.\mathrm{n}^{\circ} 28\right)$

1 La vingt-huitième parution de Changer d'époque (anciennement Les cahiers du GRHIS, Groupe de Recherche d'Histoire de l'Université de Rouen) est consacrée aux tabellionages normands. Le but de cet ouvrage collectif est de présenter cette riche source que constituent les registres de tabellionage qui sont nombreux à être conservés en Normandie. Le sous-titre ("Un notariat à découvrir ») indique que cette publication doit être vue par les chercheurs comme un éventail de pistes pour les guider dans le dépouillement des registres de tabellionage. Cet ouvrage est un complément régional et médiéval de l'ouvrage de synthèse Tabellions et tabellionages de la France médiévale et moderne, publié en 2011 sous la direction de Mathieu Arnoux et Olivier Guyotjeannin ; du reste, plusieurs auteurs ont participé aux deux projets. Le volume, composé de neuf articles, d'une introduction et d'une conclusion (toutes deux de Jean-Louis Roch), ainsi que d'une importante bibliographie sur le sujet, explore les différentes facettes du tabellionage normand. Au sein des sources normandes, la part belle est faite aux registres rouennais, puisque quatre études leur sont consacrées, par Philippe Cailleux, Philippe Levaudel, Alain Sadourny et Philippe Lardin. De manière générale, cet ouvrage s'attarde quasi exclusivement sur les villes plutôt que sur les campagnes; pourtant, le 
monde rural n'est pas absent des registres de tabellionage. La carte jointe à l'article de Philippe Lardin (p.33), présentant les différentes provenances des apprentis et valets cités dans le tabellionage rouennais, souligne ce phénomène, reprenant ainsi l'étude que l'auteur avait publiée en 2000 dans L'Argent du village du XIII au XVIII siècle, dirigé par Antoine Follain aux Presses universitaires de Rennes. Denise Angers souligne également l'apport du tabellionage pour les études du monde rural dans son article. Les auteurs n'hésitent pas à dépasser les bornes chronologiques et géographiques fixées, puisque Jean Thibault offre une intéressante étude comparative sur les notariats et tabellionages orléanais et nivernais, tandis que Virginie Lemonnier-Lesage s'intéresse aux actes produits aux XVI $\mathrm{I}^{\mathrm{e}}-\mathrm{XVIII}^{\mathrm{e}}$ siècles.

2 Les articles ont été regroupés selon deux axes. La première partie (intitulée : « Nature des registres et rôle des tabellions ») est une approche méthodologique consacrée à la forme des registres et à leurs conditions de production. La seconde (simplement intitulée : "De quelques domaines éclairés par les tabellionages») vise à montrer les différentes thématiques de recherches que peut explorer l'historien au moyen de ces sources. Si on peut regretter que les qualités des auteurs, leur organisme de rattachement et leur domaine d'étude ne soient pas précisés dans l'ouvrage, nombre de chercheurs s'intéressant à la Normandie médiévale auront reconnu des spécialistes de cet espace, travaillant depuis de nombreuses années sur les sources normandes. Les constatations et les pistes de recherches énoncées sont donc le fruit d'une solide et longue expérience, gage supplémentaire du sérieux et de l'intérêt de ce volume.

Le premier article, dû à Isabelle Bretthauer, permet de s'interroger sur le statut des registres de tabellionage. L'auteure questionne la mise en place du tabellionage, l'origine de cette mise en registre et l'usage qui en est fait par les différents membres de la société. Elle insiste particulièrement sur l'ambiguïté des registres : appartenant à la sphère privée, ils sont émanation et propriété du tabellion (il peut aussi s'agir de copies de lettres scellées) et possèdent donc une valeur administrative non négligeable (pièce justificative dans un procès, utilisation pour prélever les taxes sur la mutation d'héritage...) qui les rattache à la sphère publique. Leur conservation est un enjeu que développe Isabelle Bretthauer, soulignant avec pertinence leur longue durée de conservation, mais aussi la particularité de leur statut en tant que biens meubles qui les soumet aux circulations marchandes (gages, objets d'échanges). Il est appréciable que les textes cités soient intégralement transcrits en annexe, ce qui permet de visualiser le type d'actes dont il est question. En prenant appui sur les registres rouennais, Philippe Cailleux poursuit l'enquête sur les conditions de production de registre de tabellionage à travers l'étude du coût de ces actes. Ses recherches sont fondées sur l'analyse de deux coutumiers normands qui détaillent d'un point de vue normatif les droits, devoirs et tarifs du tabellion, ces derniers étant répartis en trois items : le coût du scel, celui du registre et celui de l'écriture. L'intérêt de cette étude réside dans la comparaison de ces données normatives avec les données effectivement trouvées dans les actes des tabellions (notamment en termes de tarifs). Enfin, la lecture des coutumiers (transcris en annexe) permet d'avoir un aperçu de l'exercice du métier de tabellion; les conditions "théoriques» du passage de l'acte sont ainsi précisées: présence de témoins, bonne compréhension des différentes parties, état de "lucidité " des contractants, lieu de passage de l'acte. Avec cette confrontation de sources (coutumiers versus registres du tabellionage), Philippe Cailleux offre un intéressant point de comparaison illustrant les conditions de productions des registres rouennais. 
4 Si l'introduction de Jean-Louis Roch rappelle à juste titre que l'on a souvent opposé une France du Sud, royaume des notaires, à celle du Nord, domaine des tabellions, la réflexion menée par Jean Thibault sur les tabellions et notaires d'Orléans et de Nevers montre combien cette dichotomie est contestable et peut s'avérer dans la région ligérienne peu pertinente. Jean Thibault s'interroge en effet sur les modalités d'exercice de ces activités ainsi que sur les liens et les différences entre ces deux professions. À Nevers, les deux professions coexistent et ont des prérogatives semblables qui vont jusqu'à la confusion. Il s'agit donc de souligner que la différence entre notaires et tabellions est loin d'être évidente dans certaines régions.

5 Enfin, la dernière étude de cette partie est consacrée à l'expression du régime communautaire des époux dans les actes des tabellions et des notaires de la Normandie coutumière de l'époque moderne. Cette recherche part d'un constat. D'après la coutume normande, il ne peut pas y avoir de communauté de biens entre les époux ; un article du coutumier précise même que cet état de fait ne peut être modifié par accord ou contrat entre les parties. Or, Virginie Lemonnier-Lesage relève que des époux ont essayé d'esquiver cette interdiction. En effet, en s'appuyant sur les formulations trouvées dans les actes de la pratique, elle démontre la virtuosité et la subtilité des notaires et tabellions afin de contourner « légalement » cette interdiction et permettre aux époux le souhaitant la mise en place d'une communauté de bien ; elle souligne par là même leur connaissance de la coutume et du droit.

6 La seconde partie de l'ouvrage regroupe des communications qui ne s'intéressent plus à la forme, mais aux contenus des actes et à leurs riches possibilités pour l'historien. Tout en soulignant les lacunes de conservation des registres de tabellionage (dont seuls ceux correspondant aux héritages sont conservés par ailleurs), Denise Angers choisit d'étudier deux périodes précises de l'histoire de Caen (1381-1382 et 1460-1475) et se concentre essentiellement sur les différents contrats mis en œuvre dans le registre: contrats de prêt, baux à cheptel et baux à ferme pour le xIV siècle, achats immobiliers, achats de rente et contrat de fieffe pour la seconde moitié du $\mathrm{Xv}^{\mathrm{e}}$ siècle. L'auteure démontre l'utilité de ces actes pour la connaissance des acteurs sociaux, tout en soulignant l'importance de construire des bases de données afin d'exploiter au mieux le tabellionage. Des acteurs sociaux, il en est question dans l'étude de Philippe Levaudel consacrée aux conseillers de Rouen présents dans le tabellionage. L'intérêt principal de cette analyse est son approche méthodologique pour identifier les conseillers rouennais, fondée sur la confrontation entre les sources municipales, l'étude des noms et des épithètes et les paroisses d'origines. Philippe Levaudel illustre ainsi les possibilités offertes par le tabellionage pour reconstituer des généalogies et étudier les milieux sociaux ; mais son étude ne s'arrête pas là et souligne l'usage qui peut être fait de ces sources dans l'estimation de la fortune (immobilière) des familles. Bruno Sintic, quant à lui, démontre que l'étude des milieux sociaux n'est pas uniquement possible pour les villes importantes, car il existe aussi des registres de tabellionage conservés pour les petites villes normandes, comme Louviers, Vernon, Montivilliers ou PontAudemer. Il met l'accent sur différents éléments repérables dans les registres, tels les milieux sociaux (bourgeois, officiers et gens de justice et même prêtre), ou les mécanismes économiques, telles les dispositions prises par des personnes âgées pour garantir leurs vieux jours, l'endettement ou la circulation et redistribution des richesses en ville. Toutefois, ainsi que le montre très brièvement Alain Sadourny en deux pages, le tabellionage n'est pas une source très riche pour l'étude des transactions 
commerciales. La dernière étude, celle de Philippe Lardin, s'intéresse aux contrats d'apprentissage et d'allouements encore présents dans les registres de tabellionage à la fin $d u X v^{e}$ et au début du Xve siècle. Son analyse fine de ces 74 actes est un excellent exemple des informations que l'on peut tirer des sources pour mieux connaître les modalités de passage du contrat, le prix de l'apprentissage, sa durée, son but et même ses conditions de résiliation. La même analyse est appliquée aux contrats d'allouement, offrant ainsi un bel aperçu de certains aspects du travail dans la région rouennaise.

7 De cet ouvrage, dont on ne peut que regretter la brièveté au vu de l'intérêt et des riches pistes de réflexion proposées, nous soulignerons l'importance de la bibliographie (plus de dix pages), qui met en avant de nombreuses études locales sur les tabellionages, mais aussi des études plus vastes fondées en partie sur l'étude de registres de tabellionage, ce que leur titre ne laisserait pas toujours deviner. Le chercheur dispose ainsi de vastes possibilités de comparaison. Mettons, au rang des regrets, l'absence d'illustrations permettant au lecteur de se rendre compte par lui-même à quoi ressemble un registre de tabellionage dans sa matérialité. Par la diversité des approches tant méthodologiques que formelles, analytiques ou thématiques, cet ouvrage atteint ainsi largement ses objectifs didactiques et offre une belle présentation de ce que sont les registres de tabellionage dont l'exploration reste souvent ardue. 